\title{
DIVERSIDAD DIFERENCIAL DE ESPORAS DE GLOMEROMYCOTA EN LA RIZOSFERA DE BROMELIÁCEAS NATIVAS DEL PARQUE NACIONAL SIERRA DE LAS QUIJADAS (SAN LUIS, ARGENTINA)
}

\author{
M. S. RIVERO MEGA'1 , E. M. CRESPO ${ }^{1}$, M. G. MOLINA² y M. A. LUGO'1,3
}

\begin{abstract}
Summary: Differential diversity of Glomeromycota in relation to host species in the rhizosphere of native bromeliads from Sierra de las Quijadas National Park (San Luis, Argentina). Arbuscular mycorrhizal fungi (AMF) are associated with most of the plants, forming arbuscular mycorrhizas. Bromeliaceae is an American family living from the tropics to temperate and arid zones. Sierra de las Quijadas has species of terrestrial Bromeliaceae colonized by AMF with different degree of association. The aim of this work was to study the AMF spore diversity (richness and density) in the rhizospheric soil of three native species of Bromeliaceae from Sierra de las Quijadas. Richness and density of spores differed significantly between the Bromeliaceae species studied, with the lowest values in Bromelia urbaniana. Meanwhile, Deuterocohnia longipetala and Dyckia floribunda showed the highest values and only differed between them in richness of spores. This AMF differential diversity between the three bromeliads species may be related to the host species and also due to different levels of root colonization that were previously reported. Moreover, the low richness and density of AMF in the rhizosphere of $B$. urbaniana may be either due to an allelopathic effect of this plant or due to its particular root morphology.
\end{abstract}

Key words: native arbuscular mycorrhizal fungi, richness, density, Bromeliaceae, host species.

Resumen: Los hongos micorrícicos arbusculares (HMA) forman con la mayoría de las plantas asociaciones simbióticas denominadas micorrizas arbusculares. Bromeliaceae es una familia americana que habita desde los trópicos hasta zonas templadas y áridas. En el Parque Nacional Sierra de las Quijadas (San Luis, Argentina) las especies terrestres de Bromeliaceae están colonizadas por HMA, presentando distintos grados de asociación. El objetivo de este trabajo fue estudiar la diversidad (riqueza y densidad) de esporas de HMA en el suelo rizosférico de tres especies nativas de Bromeliaceae del Parque Nacional Sierra de las Quijadas. Riqueza y densidad de esporas difirieron significativamente entre las especies de Bromeliaceae estudiadas, siendo siempre marcadamente menores los valores obtenidos en Bromelia urbaniana, mientras que Deuterocohnia longipetala y Dyckia floribunda presentaron los mayores valores y sólo difirieron entre sí en la riqueza de HMA. Esta diversidad diferencial de esporas de HMA observada, podría tener relación con la especie hospedante y a su vez, con los distintos grados de colonización radical de cada especie que fueron descriptos en trabajos anteriores. Además, la escasa riqueza y densidad de esporas de HMA en $B$. urbaniana podría deberse tanto a un efecto alelopático de esta planta como a su particular morfología radical.

Palabras clave: hongos micorrícicos arbusculares nativos, riqueza, densidad, Bromeliaceae, especie hospedante.

\section{INTRODUCCIÓN}

Las micorrizas son asociaciones simbióticas establecidas entre determinados grupos de hongos y las raíces de plantas vasculares y órganos de

\footnotetext{
${ }_{2}^{1}$ Diversidad Vegetal I, Área Ecología, FQBYF-UNSL

${ }^{2}$ Morfología Vegetal, Área Ecología, FQBYF-UNSL

${ }^{3}$ IMIBIO SL-CONICET

Autor para correspondencia: solemega@live.com
}

absorción de Bryophyta s.l. En general, en estas simbiosis, los hongos reciben de los hospedantes compuestos carbonados, hábitat y diversidad de nichos ecológicos, mientras que les entregan a las plantas nutrientes, especialmente las sustancias poco móviles o de baja disponibilidad en el suelo (como el fósforo $\mathrm{y}$ el nitrógeno), minerales $\mathrm{y}$ agua. Además, brindan al hospedante algunos beneficios, tales como producción de sustancias reguladoras del crecimiento, aumento de la taza fotosintética y ajuste 
osmótico a la sequía, entre otros. Aproximadamente, el $90 \%$ de las plantas posee algún tipo de micorriza, de las que el $80 \%$ son micorrizas arbusculares (MA), formadas por hongos micorrícicos arbusculares (HMA), todos ellos representantes de Glomeromycota (Brundrett, 2009; Peterson et al., 2004; Smith \& Read, 2008).

Los Glomeromycota se caracterizan por su reproducción asexual, por ser simbiontes biótrofos obligados y por formar MA. Si bien las esporas de resistencia, junto con el micelio extra-radical y los fragmentos de raíces colonizadas, son los propágalos que pueden reiniciar la colonización de los hospedantes, son solo las esporas las que permanecen viables durante largos períodos de tiempo en el suelo, constituyendo la mejor fuente definida de inóculo. Además, son los únicos propágalos de HMA que pueden identificarse a nivel específico con cierto grado de certeza (Smith \& Read, 2008). La taxonomía tradicional de los Glomeromycota permite delimitar morfo-especies y está basada en las características morfo-anatómicas de las esporas (cantidad y tipos de paredes, ornamentaciones, coloración, tamaño, forma, etc.) y en el tipo de desarrollo de las mismas (Schüßler \& Walker, 2010; Smith \& Read, 2008). Actualmente, teniendo en cuenta predominantemente las características moleculares y morfo-anatómicas de las esporas, los HMA están representados por ca. 230 especies incluidas en Glomeromycota (Krüger et al., 2011; Redecker et al., 2013; www.amfphylogeny.com).

Bromeliaceae es una familia que agrupa 50 géneros y ca. 3.000 especies. Está representada por xerófitas mayoritariamente americanas, con distintos hábitos (epífitas, terrestres, epilíticas), son generalmente de forma arrosetada y se distribuyen en los trópicos, subtrópicos y regiones templadas. Estas plantas herbáceas presentan principalmente metabolismo crasuláceo, aunque algunas especies también pueden tener patrones fotosintéticos del tipo $\mathrm{C}_{3}$ (Benzing, 2000; Crayn et al., 2004; Haro-Carrion, 2004) y presentar características morfológicas del tipo $\mathrm{C}_{4}$ (Martin, 1994). En Argentina, Bromeliaceae cuenta con 13 gé neros y 110 especies, 42 de ellas endémicas (Zuloaga et al., 1999), hay terrestres y epífitas y tiene representantes en el Parque Nacional Sierra de las Quijadas (Del Vitto et al., 2001).

Los HMA son componentes importantes de la diversidad de los ecosistemas. Tradicionalmente, su escasa diferenciación morfológica y su amplio rango de especies hospedantes (pertenecientes a grupos muy diferentes de plantas terrestres), contribuyó a que fueran considerados como simbiontes noespecíficos. Sin embargo, ha sido demostrado el efecto de la especie hospedante sobre la riqueza y abundancia de esporas de HMA y sobre la colonización radical, permitiendo afirmar la hipótesis de "especificidad ecológica" sugerida por Harley \& Smith en 1983 (Chagnon et al., 2013; Smith \& Read, 2008). Asimismo, factores medioambientales también mostraron su influencia sobre la diversidad de los HMA y apoyaron la hipótesis de "especificad edáfica" (Kivlin et al., 2011; Veresoglou et al., 2013). Tanto la "especificidad ecológica" como la "edáfica", podrían interpretarse más como una expresión de ajustes de compatibilidades planta-HMA (debidas a d istintas presiones de selección) que a una especificidad en sentido estricto o gen-a-gen, como en las relaciones parásitas (Morton et al., 2004). Las MA son muy importantes en sistemas áridos como los que predominan en la provincia de San Luis y, en particular, en el Parque Nacional Sierra de las Quijadas. En estos ecosistemas los suelos son pobres en nutrientes, condiciones que favorecen la formación de MA como estrategia para superar el estrés impuesto por el ambiente (Read, 1991). Por otro lado, la estacionalidad y otros factores abióticos (como la concentración de minerales en el suelo) afectan a las comunidades de HMA modificando la tasa de crecimiento, la duración de la dormición y palatabilidad de las esporas, entre otros aspectos. A la par, los HMA esporulan diferencialmente y presentan cambios en la dominancia dependiendo de la especie hospedante a la que se encuentran asociados (Bever et al., 2001). Además, la abundancia de una especie de HMA es considerada como un buen indicador de su importancia ecológica (Morton et al., 2004). En conjunto, los factores mencionados cumplirían un rol crucial en la composición de las comunidades de los Glomeromycota y en el mantenimiento de su diversidad (Bever et al. 1996, 2001). Así, los factores bióticos (especie del hospedante, tipo fotosintético, ciclo de vida, etc.) y abióticos (temperatura, estacionalidad, tipo de suelo, disrupción mecánica, etc.), también pueden afectar la riqueza y abundancia de los HMA (Smith \& Read, 2008). En ambientes naturales de Argentina, existen registros de las variaciones estacionales y altitudinales en relación con los tipos fotosintéticos, grado de dominancia y ciclos de vida de hospedantes nativos, para ecosistemas semiáridos y áridos del Centro del país (jarillales y pastizales de altura) y 


\section{S. Rivero Mega et al. - Glomeromycota en Bromeliáceas de San Luis}

para la Puna (Lugo \& Cabello, 2002; Lugo et al., 2005, 2008).

Estudios previos realizados en el Parque Nacional Sierras de las Quijadas, demostraron que existen diferencias significativas en la colonización MA entre las distintas especies de bromeliáceas terrestres nativas (Lugo et al., 2009). El objetivo de este trabajo fue estudiar la diversidad (riqueza y densidad) de esporas de HMA en el suelo rizosférico de tres especies de Bromeliaceae nativas terrestres del Parque Nacional Sierra de las Quijadas, de colonización MA conocida (Lugo et al. op. cit.).

\section{MATERIALES Y MÉTOdos}

Lugar de estudio

El Parque Nacional Sierras de las Quijadas (PNSQ) está situado en el NO de la provincia de San Luis (entre $32^{\circ} 20^{\prime}, 32^{\circ} 47^{\prime}$ Lat. S y $10^{\circ} 67^{\prime}, 66^{\circ} 58^{\prime}$ Long. O), a $800 \mathrm{~m}$ etros sobre el nivel del mar (msnm) y posee una extensión de 150.000 hectáreas. Es una región árida, ubicada entre las provincias biogeográficas de Monte y Chaco (Cabrera, 1976). Las precipitaciones son escasas e i rregulares, principalmente estivales, con una media anual de $200 \mathrm{~mm}$, presentando los valores más altos (hasta $250 \mathrm{~mm}$ ) entre diciembre y enero y los mínimos (hasta $0 \mathrm{~mm}$ ) en la estación seca (de febrero a noviembre). Durante el verano las temperaturas alcanzan un valor máximo de $45^{\circ} \mathrm{C}$, mientras que en invierno llegan a un mínimo de $-12{ }^{\circ} \mathrm{C}$, existiendo grandes amplitudes térmicas diarias y anuales (Rivarola, 1997; Capitanelli, 1989). En el Parque, el agua y el viento erosionan las arcillas y areniscas del suelo, formando un paisaje peculiar llamado "Huayquerías" o "malas tierras". La flora es rica en especies xerófitas, entre las que Bromeliaceae es dominante, con un total de 10 especies nativas (Del Vitto et al., 2001).

\section{Recolección de muestras}

El muestreo se realizó durante la estación seca, en abril del 2007. Se recolectaron los suelos rizosféricos de 5 individuos por cada especie hospedante y se conservaron en freezer hasta su procesamiento. Se obtuvo el peso seco (PS) de los suelos antes de su tratamiento por tamizado húmedo y decantación (Gerdemann \& Nicolson, 1963) y centrifugación con sacarosa y cuantificación (Brundrett et al., 1996; McKenney \& Lindsey, 1987; Lugo y Cabello, 2002).

\section{Especies hospedantes}

Se analizaron tres especies de Bromeliaceae nativas del PNSQ, de las cuales se conocía previamente su colonización MA (Lugo et al., 2009): Deuterocohnia longipetala (Baker) Mez (63,5\% de colonización MA) y Dyckia floribunda Griseb. (84,5 \% de colonización MA), sin diferencias significativas entre ambas especies y Bromelia urbaniana (Mez) L. B. Sm. (sin colonización).

Las tres especies estudiadas son integrantes de la comunidad vegetal ubicada a $822 \mathrm{msnm}$ y $32^{\circ} 29^{\prime} 56.3^{\prime \prime}$ Lat. S, $67^{\circ} 0^{\prime} 34.3^{\prime \prime}$ Long. O. Las especies vegetales dominantes en esta comunidad son Aspidosperma quebracho-blanco, Bromelia urbaniana, Bulnesia retama, Larrea cuneifolia, L. divaricada, Prosopis flexuosa, Tillandsia sp, Zuccagnia punctata y algunas poáceas (Del Vitto et al., 1994). En el sitio de muestreo en particular, formaron parte de la c omunidad vegetal: Acacia furcatispina, Adesmia trijuga, Allenrolfea vaginata, Atriplex argentina, A. lampa, Baccharis latifolia, Cyclolepis genistoides, Deuterocohnia longipetala, Digitaria californica, Dyckia floribunda, Echinopsis leucantha, Gomphrena pulchella, Neobouteloua lophostachia, Opuntia sulphurea, Pappophorum caespitosum, Plectocarpa tetracantha, Schinus fasciculata, Setaria cordobensis, Sporobolus phleoides, S. pyramidatus, Tephrocactus articulatus, T. articulatus var. oligocanthus y Trichloris crinita (Fig. 1).

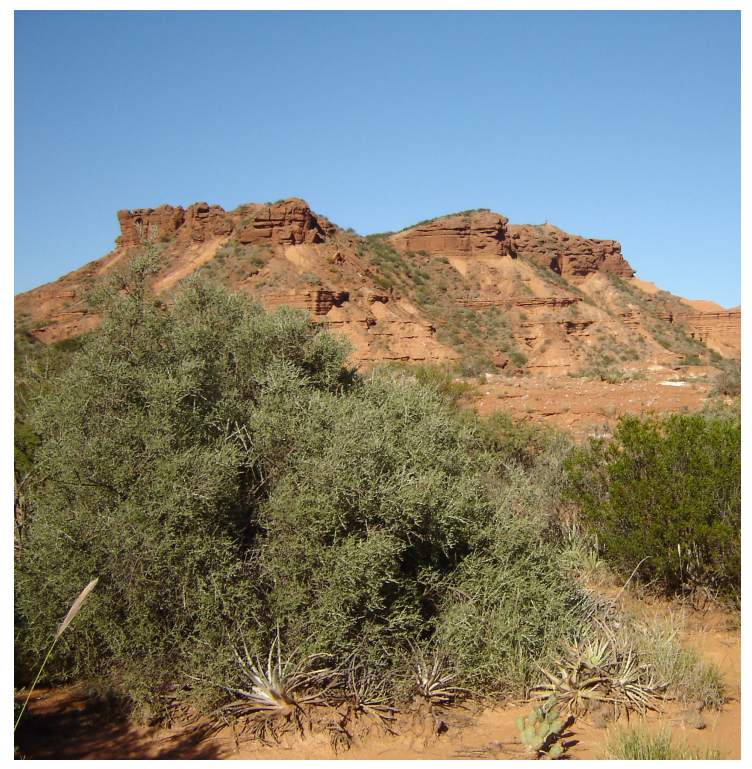

Fig. 1. Comunidad vegetal estudiada en el Parque Nacional Sierra de las Quiiadas. 


\section{Metodología}

Los suelos rizosféricos recolectados fueron secados durante 72 hs. a $70{ }^{\circ} \mathrm{C}$ en estufa de calor forzado y posteriormente tamizados utilizando el método de tamizado húmedo y decantación (Gerdemann \& Nicolson, 1963). Estas muestras fueron centrifugadas en solución de sacarosa al $50 \%$ $(\mathrm{P} / \mathrm{V})$, siguiendo la metodología de Brundrett et al. (1996). Luego se realizó la cuantificación con lupa (a 40x) de las esporas y esporocarpos extraídos, en cajas de Petri de $9 \mathrm{~cm}$ de diámetro con cuadrículas de $1 \mathrm{~cm}^{2}$ (McKenney \& Lindsey, 1987; Lugo y Cabello, 2002). Cabe mencionar que los esporocarpos fueron contados como una unidad, sin considerar el total de esporas que contenían. La caracterización morfológica de los HMA obtenidos por centrifugación se estableció siguiendo las descripciones del International Culture Collection of Vesicular Arbuscular Mycorrhizal Fungi (INVAM, http://invam.caf.wvu.edu/index.html) y Schenck \& Pérez (1990). Los taxones de Glomeromycota se clasificaron según la propuesta del Mycobank (http://www.mycobank.org/) y Schuessler Neue Seite (http://www.lrz.de/ schuessler/amphylo/).

Para evaluar la diversidad (Lewis, 2001) de Glomeromycota se consideró la densidad (densidad total de esporas $/ 100 \mathrm{~g}$ de PS de suelo rizosférico) y la riqueza absoluta (número de taxones/100g de PS de suelo rizosférico). Todos los valores de densidad y riqueza se refirieron a $100 \mathrm{~g}$ de peso seco de suelo rizosférico.

Análisis estadístico

Las variables dependientes (riqueza y densidad) se analizaron utilizando el programa INFOSTAT 2008 (actualización 2011). Previamente, se verificaron los supuestos de normalidad aplicando los tests de Shapiro Wilkins (modificado) y Levene. Así, la variable riqueza se analizó por ANAVA y la densidad por Kruskal-Wallis, considerando al taxón como variable de clasificación. Además, se aplicaron test a posteriori de Tukey para ANAVA y de comparaciones múltiples para la prueba de Kruskal-Wallis. Todos los análisis se realizaron con $\alpha=0.05$.

\section{Resultados}

La riqueza máxima de morfo-especies de Glomeromycota fue para el suelo rizosférico de $D$. longipetala (4,83/100 g de PS de suelo rizosférico). En general, los valores para esta variable fueron bajos y fluctuaron entre $1 \mathrm{y} 4,83$, variando significativamente entre las especies estudiadas $(\mathrm{F}=$ $30,42 ; \mathrm{P}=0,0001)$. La riqueza de HMA fue de $1 \mathrm{a}$ 1,5 (media=1,13) para B. urbaniana, de 3,5 a 4,83 (media $=4,19)$ para $D$. longipetala y de 1,5 a 3,49 (media $=2,27$ ) para $D$. floribunda. El test $a$ posteriori de Tukey mostró diferencias significativas entre las especies analizadas (Fig. 2).

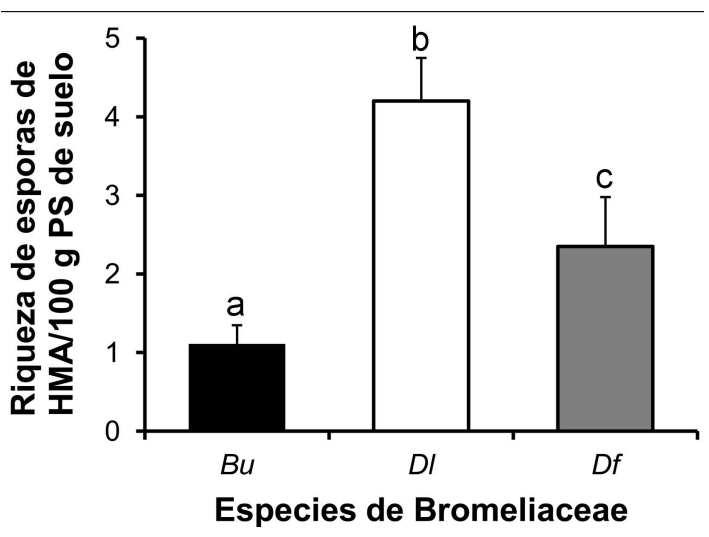

Fig. 2. Riqueza de esporas de Glomeromycota (HMA) en la rizosfera de Bromelia urbaniana (Bu), Deuterocohnia longipetala (DI) y Dyckia floribunda (Df). Los datos corresponden a I as medias de la Riqueza de esporas de HMA/100 g de PS del suelo \pm el desvío estándar. Las diferencias significativas del test a posteriori se indican con letras distintas ( $a, b$, c).

La densidad de las morfo-especies fue baja y mostró diferencias significativas entre las especies estudiadas $(\mathrm{H}=7,3 ; \mathrm{P}=0,00258)$. El valor máximo de densidad fue para $D$. floribunda: $164,41 / 100 \mathrm{~g}$ de PS de suelo rizosférico. Las densidades de esporas de HMA fueron de 9 a 24,12 (media $=16,06)$ en $B$. urbaniana, de 42,6 a $132,48($ media $=88,83$ ) en $D$. longipetala y de 22 a 164,41 (media $=81,39$ ) en $D$. floribunda. Bromelia urbaniana difirió significativamente de $D$. longipetala y de $D$. floribunda y a su vez, entre estas dos últimas, no se encontraron diferencias significativas (Fig. 3).

La riqueza absoluta total de taxones de HMA fue nueve en las rizósferas de las tres Bromeliaceae analizadas. Se hallaron diferentes morfo-especies con esporas glomoides que se designaron como Glomus aggregatum N. C. Schenck \& G. S. Sm., Funneliformis geosporus (T. H. Nicolson \& Gerd.) C. Walker \& Schüßler y Glomus sp.l. También se encontraron representantes acaulosporoides de Acaulospora sp., Acaulospora brasiliensis (B. T. Goto, L. C. Maia \& Oehl) C. Walker, M. Krüger \& 


\section{S. Rivero Mega et al. - Glomeromycota en Bromeliáceas de San Luis}

A. Schüßler y Acaulospora denticulata Sieverd. \& S. Toro y con esporas entrofosporoides, Entrophospora infrequens (I. R. Hall) R. N. Ames \& R. W. Schneid. Asimismo, se registraron Dentiscutata nigra (J. F. Redhead) Sieverd., F. A. Souza \& Oehl y Scutellospora $s p_{l}$. Además, las esporas de los HMA estuvieron frecuentemente endoparasitadas por Glomus microaggregatum Koske, Gemma \& P. D. Alexia.

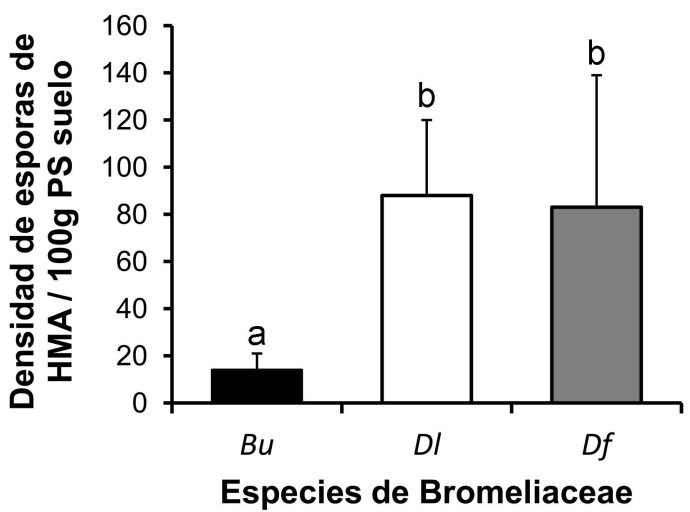

Fig. 3. Densidad de esporas de Glomeromycota (HMA) en la rizosfera de Bromelia urbaniana $(\mathbf{B u})$, Deuterocohnia longipetala (DI) y Dyckia floribunda (Df). Los datos corresponden a las medias de la Densidad de esporas de HMA/100 g de PS del suelo \pm el desvío estándar. Las diferencias significativas del test a posteriori se indican con letras distintas (a, b).

\section{Discusión Y CONCLUSIONES}

La diversidad de esporas de los HMA en el suelo rizosférico de las Bromeliaceae estudiadas (Bromelia urbaniana, Deuterocohnia longipetala y Dyckia floribunda) difirió significativamente entre las especies hospedantes. La riqueza en particular, fue diferente en las tres especies analizadas, presentando el menor valor $B$. urbaniana, el mayor $D$. longipetala y uno intermedio $D$. floribunda. En cuanto a la densidad de esporas, B. urbaniana presentó el menor valor, difiriendo significativamente de $D$. longipetala y $D$. floribunda, especies que presentaron los mayores valores. Esta diversidad diferencial de esporas de los HMA en las especies analizadas, podría sugerir la "especificidad ecológica" de la simbiosis HMABromeliaceae terrestres del PNSQ. Los conteos de esporas de los HMA constituyen un valioso acercamiento al fitness de estos hongos, ya que expresan su capacidad reproductiva, su tasa de crecimiento poblacional e indirectamente su biomasa (Bever et al., 2002). Así, la diversidad de los HMA cuantificada por sus esporas (densidad y riqueza), puede utilizarse como medida de las variaciones de las comunidades de HMA en relación a distintos factores, tales como la d iversidad de las comunidades vegetales, el tipo funcional del hospedante, la estacionalidad, la te mperatura, las características físico-químicas del suelo (Bever et al. 2002) y, en particular, la especie hospedante (Bever et al., 1996; Chagnon et al., 2013; Eom et al., 2000; Johnson et al., 1992; Sanders \& Fitter, 1992). Los resultados obtenidos de los HMA-Bromeliaceae terrestres del PNSQ, refuerzan la hipótesis de "especificidad ecológica", que también ha sido mencionada para otros ecosistemas semiáridos y áridos de jarillales y pastizales de altura del Centro de Argentina (Lugo \& Cabello, 2002; Lugo et al., 2005).

La riqueza de esporas de los HMA en el suelo rizosférico de las Bromeliaceae del PNSQ fue baja. A nivel mundial, este parámetro varía con los tipos ecológicos, la humedad ambiente, la especie hospedante, los tipos de suelo y el grado de perturbación de los ecosistemas, entre otros factores. En ambientes áridos y perturbados, la diversidad de los HMA g eneralmente es escasa (Morton et al., 2004; Walker et al., 1982; Oehl et al., 2010). En Argentina, en ambientes naturales áridos y semiáridos del Dominio Chaqueño y la Puna (Lugo et al., 2005; Lugo et al., 2008), ya fueron mencionados valores de riqueza de esporas de HMA similares al os obtenidos, mientras que fueron menores que los hallados en otros ecosistemas del país, como pastizales de altura y bosques de Polylepis australis de la Pampa de Achala y Sierras Grandes (Lugo \& Cabello, 2002; Menoyo et al., 2009), ambientes serranos de Bosque Chaqueño (Grilli et al., 2012; Urcelay et al., 2009), Salinas Grandes y Salinas de Ambargasta (Soteras et al., 2012), planicies de glaciares fueguinos (Mendoza et al., 2011) y palmares de Entre Ríos (Velázquez, 2010).

También fue escasa la densidad de esporas de los HMA asociadas a las Bromeliaceae del PNSQ. Los valores obtenidos fueron menores a los registrados para diversos ecosistemas nativos de distintas regiones fitogeográficas del país (Fontenla et al., 2001; Grilli et al., 2012; Lugo \& Cabello, 2002; Lugo et al., 2005, 2008; Menoyo et al., 2009; 
Schroeder, 2009; Soteras et al., 2012; Velázquez, 2010; Urcelay et al., 2009) y mayores a los registrados para zonas de glaciares de Tierra del Fuego (Mendoza et al., 2011). Así, los bajos valores de riqueza y densidad de esporas de HMA en los suelos rizosféricos de bromeliáceas en Sierras de las Quijadas, sugieren lo poco propicio de este ambiente para la proliferación de los HMA.

Glomus s. $l$. fue el género de HMA más frecuentemente hallado en las bromeliáceas estudiadas del PNSQ. Los disturbios pueden alterar la composición de las comunidades de HMA y también disminuir su diversidad; por ejemplo, como consecuencia de las perturbaciones de origen agronómico, puede predominar Glomus sobre Gigaspora y Scutellospora, no sólo en la densidad de esporas en el suelo rizosférico del hospedante sino también en la colonización radical (Oehl et al., 2010; Smith \& Read, 2008; Torrecillas et al., 2012). Esta predominancia de Glomus bajo condiciones extremas ya se ha observado también en ecosistemas áridos y semiáridos (Jacobson, 1997), junto con especies de Entrophospora y Acaulospora, todos con representantes de esporas pequeñas (Morton et al., 2004 y referencias allí citadas). En nuestro país, tanto la predominancia de Glomus como la presencia de Entrophospora y Acualospora ya fue registrada para la Puna, jarillales (Lugo et al., 2005, 2008) y ahora en el presente trabajo.

Además, la abundancia y riqueza de esporas de los HMA en el suelo pueden ser buenos indicadores del fitness de estos hongos dentro de las raíces de algunos hospedantes (Bever et al., 2002), de tal manera que la diversidad diferencial de los HMA hallada en el suelo rizosférico de las Bromeliaceae analizadas, podría relacionarse con sus distintos grados de colonización radical. Según estudios realizados por Lugo et al. (2009), se conoce que la colonización micorrícica arbuscular es diferente entre las Bromeliaceae terrestres estudiadas: Deuterocohnia longipetala tiene un 63,5 \% de colonización, Dyckia floribunda un 84,5 \% y Bromelia urbaniana no está colonizada. Asimismo, estas especies también son diferentes en cuanto a la morfología de sus sistemas radicales: Deuterocohnia longipetala y Dyckia floribunda presentan raíces gruesas con pelos absorbentes escasos o ausentes y por el contrario, las raíces de Bromelia urbaniana son finas, ramificadas y con abundantes pelos radicales (Lugo com. pers.). Es bien conocido que la morfología radical del hospedante se relaciona con el grado de asociación micorrícica y la capacidad de absorber nutrientes; Baylis, en 1975 (ver Fitter,
2004), propuso que las plantas con sistemas radicales poco desarrollados estarían obligadas a asociarse simbióticamente para poder nutrirse, mientras que lo contrario se esperaría en aquellas plantas con sistemas radicales muy desarrollados (con raíces finas y abundantes pelos radicales), que les permiten explorar más eficientemente el suelo para la captura de nutrientes (Brundrett, 2002). Por lo tanto, las raíces de $D$. longipetala y $D$. floribunda cumplirían con las características típicas de plantas con alto grado de colonización micorrícica, mientras que $B$. urbaniana correspondería a las plantas con escasa colonización micorrícica o no micorrícicas. Así, los mayores valores de abundancia y riqueza de los HMA en las rizósferas de $D$. longipetala y $D$. floribunda en relación con $B$. urbaniana, se corresponden con el mayor o menor fitness de los HMA dentro de las raíces de estos hospedantes (Bever et al., 2002).

Por otra parte, la escasa riqueza y densidad de esporas de HMA en el suelo rizosférico de $B$. urbaniana podría deberse además a un efecto alelopático de esta planta sobre los HMA. La planta podría repeler a los HMA como una posible estrategia para evitar la colonización, ya que la misma redundaría en pérdidas energéticas del hospedante, debido al consumo de fotosintetato por parte del simbionte fúngico (Johnson \& Graham, 2013). Asimismo, se conocen efectos citotóxicos en otras especies del género Bromelia (Raffauf et al., 1981), debido a la producción de flavonoides, compuestos que afectan la colonización por HMA (Harrison, 1997). Esta alelopatía podría también influir negativamente en la diversidad (riqueza y densidad) de los HMA en la rizosfera de $B$. urbaniana, lo que determinaría su nula colonización radical. Los compuestos químicos producidos en las raíces son claves para entender la fisiología de la exclusión de los HMA en las plantas no micorrícicas (Brundrett, 2002). Por lo tanto, sería necesario la realización de estudios sobre el sistema radical de $B$. urbaniana (e. g. determinar presencia o ausencia de metabolitos secundarios capaces de inhibir el crecimiento fúngico), para esclarecer el mecanismo de interacción $B$. urbaniana-HMA y determinar las causas de la ausencia de estos simbiontes fúngicos en su rizosfera.

En el PNSQ, la riqueza y densidad de esporas de HMA en el suelo rizosférico de las Bromeliaceae terrestres varió significativamente entre las especies estudiadas, lo que permite reforzar la hipótesis de la especificidad ecológica entre los HMABromeliaceae nativas, evidenciando la existencia de 


\section{S. Rivero Mega et al. - Glomeromycota en Bromeliáceas de San Luis}

una relación diferencial con la especie hospedante y con el fitness de los HMA en la asociación. Además, esta diversidad diferencial de los HMA podría estar relacionada con otros factores, tales como la arquitectura radical y efectos alelopáticos en el caso de B. urbaniana.

\section{AgRAdECIMIENTOS}

Este trabajo de investigación se realizó en el marco del proyecto PROICO 2-2214, financiado por la FQByF (UNSL) y el PICT 2008-0781 financiado por la FONCyT.

\section{BIBLIOGRAFÍA}

BENZING, D. H. 2000. Introduction. In: BENZING, D.H. (eds.), Bromeliaceae: Profile of an adapt ative radiation, pp. 1-15. Cambridge University Press, Cambridge.

BEVER, J. D., J. B. MORTON, J. ANTONOVICS \& P. A. SCHULTZ. 1996. Host-dependent sporulation and species diversity of arbuscular mycorrhizal fungi in a mown grassland. $J$. Ecol. 84: 71-82.

BEVER, J. D., A. P. SCHULTZ, A. PRINGLE \& J. B. MORTON. 2001. Arbuscular mycorrhizal fungi: more diverse than meets the eye, and the ecological tale of why. BioScience 51: 923931.

BEVER, J. D., A. PRINGLE \& P. A. SCHULTZ. 2002. Dynamics within the plant-arbuscular mychorrhizal funfi mutualism: testing the nature of community feedback. In: M. G. A. VAN DER HEIJDEN \& I. R. SANDERS (eds.), Mycorrhizal Ecology, pp. 267-292. Springer, Alemania.

BRUNDRETT, M. C. 2002. Coevolution of roots and mycorrhizas of land plants. Tansley review no. 134, New Phytol. 154: 275-304.

BRUNDRETT, M. C. 2009. Mycorrhizal associations and other means of n utrition of vascular plants: understanding the global diversity of host plants by resolving conflicting information and developing reliable means of diagnosis. Plant Soil 320: 37-77.

BRUNDRETT, M. C., N. BOUGHER, B. DELL, T. GROVE \& N. MALAJCZUK. 1996. Working with mycorrhizas in forestry and agriculture. CSIRO, Australia.
CABRERA, A. L. 1976. Territorios fitogeográficos de la República Argentina. En: Enciclopedia Argentina de Agricultura y Jardinería, pp. 185. 2da. ed. ACME, Buenos Aires.

CAPITANELLI, R. 1989. Geografia de San Luis. Gobierno de la Prov. de San Luis. Ministerio de Cultura y Educación, pp. 205-216.

CHAGNON, P. L., R. L. BRADLEY, H. MAHERALI \& J. N. KLIRONOMOS. 2013. A trait-based framework to understand life history of mycorrhizal fungi. Trends Plant Sci. 18: 484-491.

CRAYN, M., K. WINTER \& A. C. SMITH. 2004. Multiple origins of crassulacean acid metabolism and the epiphytic habit in the Neotropical family Bromeliaceae. Proc. Natl. Acad. Sci. U S A 101: 3703-3708.

DEL VitTO, L. A., E. M. PETENATTI, M. M. NELLAR \& M. E. PETENATTI. 1994. Las áreas protegidas de San Luis, Argentina. Multequina 3: 141-156.

DEL VITTO, L. A., E. M. PETENATTI \& M. E. PETENATTI. 2001. Catálogo preliminar de la flora vascular. Parque Nacional Sierras de las Quijadas, San Luis, Argentina. Serie Técnica del Herbario UNSL 8, Argentina.

EOM, A. H., D. C. HARTNETT \& G. W. T. WILSON. 2000. Host plant species effects on arbuscular mycorrhizal fungal communities in tallgrass prairie. Oecologia 122: 435-444.

FITTER, A. H. 2004. Magnolioid roots - hairs, architecture and mycorrhizal dependency. New Phytol. 164: 15-16.

FONTENLA, S., N. BACCALÁ \& M. HAVRYLENKO. 2001. Dinámica de las micorrizas arbusculares en dos bosques de Austrocedrus chilensis con diferente estado sanitario. Ecología 15: 37-44.

GERDEMANN, J. W. \& T. H. NICOLSON. 1963. Spores of mycorrhizal Endogone extracted from soil by wet sieving and decanting. Trans. Brit. Mycol. Soc. 46: 235-244.

GRILLI, G., C. URCELAY \& L. GALETTO. 2012. Forest fragment size and nutrient availability: complex responses of mycorrhizal fungi in native-exotic hosts. Plant Ecol. 213:155-165.

HARO-CARRION, X. 2004. Bromeliad distribution in two plots in the Sumaco Biosphere Reserve. Lyonia 7: 57-62.

HARRISON, M.J. 1997. The arbuscular mycorrhizal symbiosis: an underground association. Trends Plant Sci. 2: 54-60. 
JACOBSON, K. M. 1997. Moisture and substrate stability determine VA-mycorrhizal fungal distribution and structure in an arid grassland. J. Arid Environ. 35:59-75.

JOHNSON, N. C., D. TILMAN \& D. WEDIN. 1992. Plant and soil controls on mycorrhizal fungal communities. Ecology 73: 2034-2042.

JOHNSON, N. C. \& J. H. GRAHAM. 2013. The continuum concept remains a useful framework for studying mycorrhizal functioning. Plant Soil 363: 411-419.

KIVLIN, S. N., C. V. HAWKES \& K. K. TRESEDER. 2011. Global diversity and distribution of arbuscular mycorrhizal fungi. Soil Biol. Biochem. 43: 2294-2303.

KRÜGER, M., C. KRÜGER, C. WALKER, H. STOCKINGER \& A. SCHÜßLER. 2011. Phylogenetic reference data for systematics and phylotaxonomy of arbuscular mycorrhizal fungi from phylum to species level. New Phytol. 183: 212-223.

LEWIS, J. P. 2001. La Biósfera y sus ecosistemas. Una introducción a la ecología. ECOSUR, Serie de Publicaciones Técnicas No2, Argentina.

LUGO, M. A. \& M. N. CABELLO. 2002. Native arbuscular mycorrhizal fungi (AMF) from mountain grassland (Córdoba, Argentina). Seasonal variation of fungal spore diversity. Mycologia 94: 579-586.

LUGO, M. A., A. M. ANTON \& M. N. CABELLO. 2005. Arbuscular mycorrhizas in the Larrea divaricata shrubland at arid "Chaco", Central Argentina. J. Agric. Tech. 1: 163-178.

LUGO, M. A., M. FERRERO, E. MENOYO, M. C. ESTÉVEZ, F. SIÑERIZ \& A. M. ANTON. 2008. Arbuscular mycorrhizal fungi and rhizospheric bacteria diversity along an altitudinal gradient in South American Puna grassland. Microb. Ecol. 55: 705-713.

LUGO, M. A., M. G. MOLINA \& E. M. CRESPO. 2009. Arbuscular mycorrhizas and dark septate endophytes in bromeliads from South American arid environment. Symbiosis 47: 1721.

GRILLI, G., C. URCELAY \& L. GALETTO. 2012. Forest fragment size and nutrient availability: complex responses of mycorrhizal fungi in native-exotic hosts. Plant Ecol. 213:155-165.

MARTIN, C. E. 1994. Physiological ecology of the Bromeliaceae. Bot. Rev. 60: 1-82.

MCKENNEY, M. C. \& D. L LINDSEY. 1987. Improved methods for quantifying endomycorrhizal fungi spores from soil. Mycologia 79: 779-782.

MENDOZA R., M. CABELLO, J. ANCHORENA, I. GARCÍA, L. MARBÁN. 2011. Soil parameters and host plants associated with arbuscular mycorrhizae in the grazed Magellanic steppe of Tierra del Fuego. Agricult. Ecosys. Environ. 140: 411-418.

MENOYO E., D. RENISON \& A. G. BECERRA. 2009. Arbuscular mycorrhizas and performance of Polylepis australis trees in relation to livestock density. Forest Ecol. Manag. 258: 2676-2682.

MORTON, J. B., R. E. KOSKE, S. L. STÜMER \& S. P. BENTIVENGA. 2004. Mu tualistic arbuscular endomycorrhizal fungi. In: MUELlER G. M., G. F. BILLS \& M. S. FOSTER (eds.), Biodiversity of Fungi. Inventory and monitoring methods, pp. 317336. Elsevier Academic Press, Amsterdan.

OEHL F., E. LACZKO, A. BOGENRIEDER, K. STAHR, R. BÖSCH, M. van der HEIJDEN \& E. SIEVERDING. 2010. Soil type and land use intensity determine the composition of arbuscular mycorrhizal fungal communities. Sol. Biol. Biochem. 42: 724-738.

PETERSON, R. L., H. B. MASSICOTTE \& L. H. MELVILLE. 2004. Mycorrhizas: anatomy and cell biology. NRC-Research Press, Ottawa.

RAFFAUF, R.F., M. D. MENACHERY, P. W. LE QUESNE, E. V. ARNOLD \& J. CLARDY. 1981. Antitumor plants. 11 diterpenoid and flavonoid constituents of Bromelia pinguin L. J. Organic Chem. 46: 1094-1098.

READ, D. J. 1991. Mycorrhizas in ecosystems. Experientia 47: 376-391.

REDECKER, D., A. SCHÜßLER, H. STOCKINGER, S. STÜRMER, J. MORTON \& C. WALKER. 2013. An evidence-based consensus for the classification of arbuscular mycorrhizal fungi (Glomeromycota). Mycorrhiza 23: 515-531.

RIVAROLA, D. 1997. El Parque Nacional Sierra de las Quijadas y sus recursos naturales. Printers Impresores, San Luis.

SANDERS, I. R. \& A. H. FITTER. 1992. Evidence for differential responses between host-fungus combinations of vesicular-arbuscular mycorrhizas from a grassland. Mycol. Res. 96: 415-419.

SCHENCK, N. C. \& Y. PEREZ. 1990. Manual for the identification of VA mycorrhizal fungi. 3rd ed. Synergistic Publications, Gainesville. 


\section{S. Rivero Mega et al. - Glomeromycota en Bromeliáceas de San Luis}

SCHROEDER, M. A. 2009. Una alternativa a los cultivos tradicionales en el NO de la provincia de Corrientes. Medición de biomasa y análisis químicos de tejidos en plantines micorrizados de Aloysia polystachya (Grisebach) Moldenke y Lippia turbinata Grisebach. Tesis de maestría, UNNE.

SCHÜßLER, A. \& C. WALKER. 2010. The Glomeromycota: a species list with new families and new genera. Libraries of The Royal Botanic Garden, Kew. Gloucester.

SMITH, S. E \& D. J. READ. 2008. Mycorrhizal symbiosis. 3rd ed. Academic Press, London.

SOTERAS F., A. BECERRA, N. COFRÉ, J. BARTOLONI \& M. CABELLO. 2012. Arbuscular mycorrhizal fungal species in saline environments of Central Argentina: seasonal variation and distribution of spores at different soil depths. Sydowia 64: 301-313.

TORRECILLAS, E., M. M. ALGUACIL \& A. ROLDÁN. 2012. Host preferences the arbuscular mycorrhizzal fungi colonizing annual herbaceous plant species in semiarid mediterranean prairies. Appl. Environ. Microbiol. 78: 6180-6186.

URCELAY C., S. DÍAZ, D. E. GURVICH, F. S. CHAPIN , E. CUEVAS \& L. S. DOMÍNGUEZ. 2009. Mycorrhizal community resilience in response to experimental plant functional type removals in a woody ecosystem. J. Ecol. 97: 1291-1301.
VELÁZQUEZ, M. S. 2010. Comunidades de hongos formadores de micorrizas arbusculares en el Parque Nacional "El Palmar". Tesis doctoral. UNLa Plata.

VERESOGLOU, S. F., T. CARUSO \& C. RILLIG. 2013. Modelling the environmental and soil factors that shape the niches of two common arbuscular mycorrhizal fungal families. Plant Soil 368: 507-518.

ZULOAGA, F. O., O. MORRONE \& D. RODRÍGUEZ. 1999. Análisis de la biodiversidad en plantas vasculares de la Argentina. Kurtziana 27: 17-167.

WALKER, C., C.W. MIZE \& H.S. McNABB. 1982. Populations of endogonaceous fungi at two populations in central Iowa. Can. J. Bot. 60: 2518-2529.

www.amf-phylogeny.com [Acceso: septiembre 2013]

http://invam.caf.wvu.edu/index.html [Acceso: septiembre 2013]

http://www.lrz.de/ schuessler/amphylo/ [Acceso: septiembre 2013]

http://www.mycobank.org/ [Acceso: septiembre 2013]

Recibido el 10 de octubre de 2013, aceptado el 19 de mayo de 2014. 